\title{
A Numerical Study on Slip Flow Heat Transfer in Micro-Poiseuille Flow using Perturbation Method
}

\author{
M. Saghafian ${ }^{1 \dagger}$, I. Saberian $^{1}$, R. Rajabi ${ }^{1}$ and E. Shirani ${ }^{2}$ \\ ${ }^{1}$ Department of Mechanical Engineering, Isfahan University of Technology, Isfahan,8415683111, Iran \\ 2 Foulad Institute of Technology, Fooladshahr, Isfahan, 8491663763, Iran
}

$\dagger$ †orresponding Author Email:saghafian@cc.iut.ac.ir

(Received August 27, 2013; accepted March 6, 2014)

\begin{abstract}
In this study, slip-flow heat transfer in a laminar, steady state, two-dimensional incompressible flow between parallel plates micro-channel is investigated numerically. A new method based on perturbation expansion for modeling of slip micro-flows is presented. Navier-stokes equations are developed by using perturbation expansions of velocity, pressure and temperature fields. Different orders of equations depending on the magnitude of Knudsen number are obtained and each set of the equations are solved. The computations are performed for micro-channels with Constant Wall Heat Flux (CWHF) and Constant Wall Temperature (CWT) boundary conditions to obtain heat transfer characteristics of gaseous flow in slip regime. The effects of compressibility and viscous dissipation are neglected in this study. The numerical methodology is based on Semi-Implicit Method for Pressure-Linked Equations (SIMPLE) method. The effects of Knudsen number and thermal creep flow on Nusselt number are numerically investigated.This study confirms that the perturbation method with different orders of Knudsen number can predict the velocity and temperature fields with good accuracy. The obtained solutions are compared with both available numerical and analytical results and good agreement is obtained.
\end{abstract}

Keywords:Heat transfer, slip-flow, micro-channel, parallel-plates, Nusselt number, perturbation expansion.

\section{NOMENCLATURE}

$\begin{array}{ll}p & \text { Specific heat at constant pressure, } \mathrm{kJ} / \mathrm{kgK} \\ v & \text { Specific heat at constant volume, } \mathrm{kJ} / \mathrm{kgK} \\ C_{f} & \text { Friction factor coefficient } \\ D_{h} & \text { Hydraulic diameter, } m \\ h & \text { Heat transfer coefficient, } W / \mathrm{m}^{2} K \\ H & \text { Distance between two plates, } m \\ k & \text { Thermal conductivity, } W / \mathrm{mK} \\ \mathrm{Kn} & \text { Knudsen number, } \lambda / \mathrm{H} \\ L & \text { Cannel length, } m \\ \mathrm{Nu} & \text { Nusselt number, } h D_{h} / \mathrm{k} \\ P r & \text { Prandtl number, } v / \alpha \\ P o & \text { Poiseuille number, } C_{f} \cdot R e \\ q_{w} & \text { Wall heat flux, } W / \mathrm{m}^{2} \\ R e & \text { Reynolds number, } \rho u_{m} D_{h} / \mu \\ T & \text { Temperature, } K \\ T_{w} & \text { Wall temperature, } K\end{array}$

\section{INTRODUCTION}

Due to the rapid developments in the electronic industry, microfabrication technologies and biomedical engineering in recent years the research interest on flow and heat transfer phenomena in microchannels is widely increased. Fabrication of small devices has increased the needs of understanding of fluid flow and heat

$\begin{array}{ll}T_{s} & \text { Fluid temperature at the wall, } K \\ T_{m} & \text { Bulk temperature of the fluid, } K \\ u_{i n} & \text { inlet velocity, } \mathrm{m} / \mathrm{s} \\ u_{m} & \text { Mean stream wise velocity, } \mathrm{m} / \mathrm{s} \\ U_{w} & \text { Dimensionless wall velocity, } u_{w} / u_{m} \\ U_{s} & \text { Dimensionless fluid velocity adjacent to } \\ & \text { the wall, } u_{s} / u_{m} \\ & \\ G r e e k \text { Symbols } \\ \theta & \text { Dimensionless temperature } \\ \boldsymbol{\lambda} & \text { Molecular mean free path, } \mathrm{m} \\ \boldsymbol{\mu} & \text { Dynamic viscosity, } \mathrm{kg} / \mathrm{ms} \\ \boldsymbol{\rho} & \text { Density, } \mathrm{kg} / \mathrm{m}^{3} \\ \boldsymbol{\sigma}_{\boldsymbol{T}} & \text { Thermal accommodation coefficient } \\ \boldsymbol{\sigma}_{\boldsymbol{v}} & \text { Momentum accommodation coefficient } \\ \mathbf{v} & \text { Kinematic viscosity, } \mathrm{m}^{2} / \mathrm{s}\end{array}$

transfer in micro-geometries. Microscale fluid flow and heat transfer behavior differs greatly from that of macroscale. Many experimental and numerical studies on fluid flow and heat transfer in micro-channels have been undertaken. It is known qualitatively that gaseous flow in a micro-channel is affected by the rarefaction (the slip on the surface), the surface roughness and the 
compressibility effects separately or simultaneously. Rarefaction takes place by either the size or pressure of a fluid system decreases resulting in a mean free path of the fluid molecules that is comparable to the characteristic length of the system itself. In such cases, discontinuities between the fluid and the solid surface, as well as other noncontinuum behavior begin to develop. The very first parameters that are affected by rarefaction are velocity slip and temperature jump. Further Kn number, the ratio of mean free path to the length characteristies of the flow, increases in the fluid systems cause requirement of higher order and more sophisticated models for sheer stress and heat flow.

Navier-stokes-based fluid dynamics solvers are often inaccurate when applied to Micro Electro Mechanical Device (MEMS). This inaccuracy stems from the calculation of molecular transport effects, such as viscous dissipation and thermal condition, from bulk flow quantities, such as mean velocity and temperature. The approximation of microscale phenomena using macroscale information fails as the characteristic length of the gaseous flow gradients (l) approaches the average distance traveled by molecules between collisions.

Four different flow regims are observed based on the value of the Knudsen number, continuum flow for $\mathrm{Kn} \leq$ 0.001 ; slip flow regime for $0.001 \leq \mathrm{Kn} \leq 0.1$; transition regime for $0.1 \leq \mathrm{Kn} \leq 10$ and free molecular regime for $\mathrm{Kn} \geq 10$ (Beskok and Kardianakis (1994)). Kn determines the degree of rarefaction and the degree of the validity of the continuum approach. As Kn increases, rarefaction effects become more important, and eventually the continuum approach breaks down. Palm (2001), Sobhan andGarimella (2001) and Obot (2002) reviewed the experimental results in the existing literature for the convective heat transfer in microchannels. Morini (2004)presents an excellent review of the experimental data for convective heat transfer in microchannels. Barron et al. (1996) and Barron et al. (1996) extended the Graetz problem to slip-flow and developed simplified relationships to describe the effect of slip-flow on the convection heat transfer coefficient. Ameel et al. (1997) analytically treated the problem of laminar gas flow in micro tubes with a constant heat flux boundary condition at the wall assuming a slip flow hydrodynamic condition and a temperature jump thermal condition at the wall. They found that the fully developed Nusselt number decreased with Knudsen number. Tunc and Bayazitoglu (2001)investigated steady laminar hydrodynamically developed flow in microtubes with uniform temperature and uniform heat flux boundary conditions using the integral technique. They included temperature jump condition at the wall and viscous heating within the medium. Aydin and Aydin and Avci (2001)studied laminar forced convective heat transfer of a Newtonian fluid in a microchannel between two parallel plate analytically. Sparrow and Line(1962) examined laminar heat transfer in microtubes under slip-flow conditions and Inman (1964)investigated laminar slip-flow heat transfer in a parallel-plate channel with uniform wall heating and cartesian a formula for calculation of Nusselt number in this condition. Also Hadjiconstantinou and Simek (2002) investigated the constant-wall-temperature convection heat transfer characteristics of a model gaseous flow in twodimensional micro and nano-channels under hydrodynamically and thermally fully developed conditions. They covered both the slip flow regime and most of the transition regime. Their results shown that the slip flow prediction is in good agreement with the DSMC results for $K n \leq 0.1$. They also show that the Nusselt number decreases monotonically with increasing Knudsen number in a fully accommodating case, both in slip flow and transition regimes.

The aim of the present study is to present a new method for modeling the slip velocity and temperature jump in slip flow regime. For this purpose we numerically investigate rarefied gas flow in a microchannel between two parallel plates. Both the CWHF and CWT thermal boundary conditions are applied at the wall. The governing equations are solved in conjunction with the velocity slip and temperature jump to account for rarefaction effects in slip flow regime. Three-term perturbation expansions of velocity, pressure and temperature are used for developing of governing equations. The equations with different orders of Knudsen number are obtained. These equations are solved using SIMPLE algorithm. We compare Poiseuille number and Nusselt number with the results obtained by others.

\section{Problem Description}

The computations are performed a micro-channel with constant heat flux (CWHF) and constant wall temperature (CWT) boundary conditions. We investigate hydrodynamically and thermally developing, steady state, laminar, incompressible twodimensional flow with constant properties. The flow of gas is considered through two horizontal parallel-plates of length $\mathrm{L}$ that are a distance $\mathrm{H}$ apart and a cartesian coordinate system as shown in Fig. 1. The effects of compressibility and viscous dissipation are neglected due to low speed flows associated with micro-channel. A Channel length (L) is chosen much larger than its height to ensure the thermally and hydrodynamically fully developed flow condition at the channel exists. As shown in Fig. 1, the velocity and temperature profiles are uniform at inlet of the channel and are based on $\operatorname{Re}=1$. For constant and uniform heat flux boundary condition, Nitrogen is used with constant properties. For constant-wall temperature (CWT) boundary condition, air with constant properties is selected as the working fluid in the channel. Also we assumed $\lambda$, is constant so Knudsen number varies by changing $\mathrm{H}$.

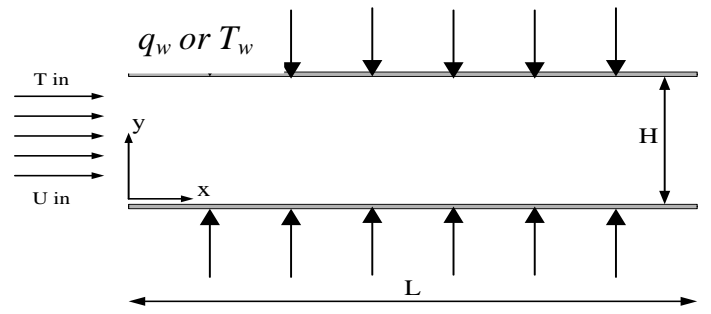

Fig. 1. Channel geometry 


\section{Perturbation Method and Numerical Solution}

The macroscopic properties $\varphi$ of the flow may be written with an asymptotic expansionas a function of the Knudsen number (Karniadakis and Beskok (2004)andQin et al. (2007)):

$\varphi=\varphi_{0}+K n \varphi_{1}+K n^{2} \varphi_{2}+K n^{3} \varphi_{3}+\ldots$

$\varphi=u, v, w, P, T, \rho, \ldots$

Where $\varphi_{0}$ corresponds to the no-slip flow, $\varphi_{1}, \varphi_{2}, \varphi_{3}$ are the first, second and third order corrections of the $\varphi_{0}$ respectively. Increase of the Knudsen number, i.e. going from slip to free molecular flow regime, higher corrections of the $\varphi$ become important. In the slip flow regime we can use Navier-Stokes equations with use appropriate velocity slip and temperature jump conditions (Karniadakis and Beskok (2004)).

In this paper, a new method for modeling of microflows is introduced. Governing equations are developed by using perturbation expansions of velocity, pressure and temperature fields. Subsequently, different orders of equations depending on the value of Knudsen number are obtained. These set of equations are discretized in two-dimensional state on a staggered grid using finite volume method. In this study three-term perturbation expansions are used. These equations are obtained for up to second order magnitude respect to $K n$. Total algorithm of solution includes three steps and solved with the SIMPLE algorithm.

Many second-order models have been proposed either experimentally or numerically and some have proven useful in increasing the range and accuracy of the slip boundary condition representation of rarefaction. There is currently insufficient experimental data to validate the use of any particular second-order model. Presently, however, there are few analytical or numerical convective heat transfer solutions based on secondorder slip boundary condition models, and these are often presented for limited values of Knudsen number, momentum and thermal accommodation coefficients, geometry and consequently have limited applicability.

In this research we use three-term expansions and substitute these expansions into momentum and energy equations and boundary conditions, Momentum and energy equations are described as:

$\nabla \cdot \vec{V}=0$

$\rho \frac{D \vec{V}}{D t}=-\nabla p+\mu \nabla^{2} \vec{V}$

$\rho C_{p} \frac{D T}{D t}=k \nabla^{2} T$

Perturbation expansions of velocity $(\vec{V}=u \hat{\imath}+v \hat{\jmath})$, pressure and temperature fields are defined by (Karniadakis and Beskok (2004)):

$u=u_{0}+K n u_{1}+K n^{2} u_{2}+\ldots$

$v=v_{0}+K n v_{1}+K n^{2} v_{2}+\ldots$

$P=P_{0}+K n P_{1}+K n^{2} P_{2}+\ldots$

$T=T_{0}+K n T_{1}+K n^{2} T_{2}+\ldots$

By substituting these expansions in Momentum and energy equations, One can reach to three-order of equations $\mathrm{O}(1), \mathrm{O}(K n), \mathrm{O}\left(K n^{2}\right)$ and their boundary conditions, therefore for the zeroth-order equations and their boundary conditions, we have:

$\rho\left[\frac{\partial \vec{V}_{0}}{\partial t}+\left(V_{0} \cdot \nabla\right) \vec{V}_{0}\right]=-\nabla P_{0}+\mu \nabla^{2} \vec{V}_{0}$

$\frac{\partial T_{0}}{\partial t}+\nabla \cdot\left(T_{0} \vec{V}_{0}\right)=\alpha \nabla^{2} T_{0}$

In fact the above equations are the no-slip NavierStokes and energy equations. The zeroth-order boundary conditions on the wall are defined as:

$\left.U_{0}\right|_{s}=U_{w}$

$\left.T_{0}\right|_{s}=T_{w}$

Where $U_{0}=u_{0} / u_{m}$ and $u_{m}$ is mean stream wise velocity. The first order $\mathrm{O}(K n)$ equations and their boundary conditions on the walls are described by:

$\rho\left[\frac{\partial \vec{V}_{1}}{\partial t}+\left(\vec{V}_{1} \cdot \nabla\right) \vec{V}_{0}+\left(\vec{V}_{0} \cdot \nabla\right) \vec{V}_{1}\right]=-\nabla P_{1}+\mu \nabla^{2} \vec{V}_{1}$

$\frac{\partial T_{1}}{\partial t}+\nabla \cdot\left(T_{0} \vec{V}_{1}\right)+\nabla \cdot\left(T_{1} \vec{V}_{0}\right)=\alpha \nabla^{2} T_{1}$

$\left.U_{1}\right|_{s}=\left.\frac{2-\sigma_{v}}{\sigma_{v}}\left(\frac{\partial U_{0}}{\partial n}\right)\right|_{s}$

$\left.T_{1}\right|_{s}=\left.\frac{2-\sigma_{T}}{\sigma_{T}}\left[\frac{2 \gamma}{\gamma+1}\right] \frac{1}{P r}\left(\frac{\partial T_{0}}{\partial n}\right)\right|_{s}$

Where $U_{l}=u_{l} / u_{m}$. Finally the second order equations $\mathrm{O}\left(\mathrm{Kn}^{2}\right)$ are presented as:

$$
\begin{aligned}
\frac{\partial \vec{V}_{2}}{\partial t}+\left(\vec{V}_{0} \cdot \nabla\right) \vec{V}_{2}+ & \left(\vec{V}_{2} \cdot \nabla\right) \vec{V}_{0}+\left(\vec{V}_{1} \cdot \nabla\right) \vec{V}_{1} \\
= & -\nabla P_{2}+\mu \nabla^{2} \vec{V}_{2} \\
\frac{\partial T_{2}}{\partial t}++\nabla \cdot\left(T_{0} \vec{V}_{2}\right)+ & \nabla \cdot\left(T_{2} \vec{V}_{0}\right)+\nabla \cdot\left(T_{1} \vec{V}_{1}\right) \\
& +\nabla \cdot\left(T_{0} U_{1}\right)=\alpha \nabla^{2} T_{2}
\end{aligned}
$$

And the boundary conditions for $\mathrm{O}\left(\mathrm{Kn}^{2}\right)$ are defined as:

$\left.U_{2}\right|_{s}=\frac{2-\sigma_{v}}{\sigma_{v}}\left[\frac{\partial U_{1}}{\partial n}+\frac{1}{2} \frac{\partial^{2} U_{0}}{\partial n^{2}}\right]$

$\left.T_{2}\right|_{s}=\frac{2-\sigma_{T}}{\sigma_{T}}\left[\frac{2 \gamma}{\gamma+1}\right] \frac{1}{P r}\left[\frac{\partial T_{1}}{\partial n}+\frac{1}{2} \frac{\partial^{2} T_{0}}{\partial n^{2}}\right]$

Where $U_{2}=u_{2} / u_{m}$. Also the first and second order equations require corrections due to the velocity-slip and temperature-jump. Total algorithm of solution includes three steps: the first step is to solve Eq. (10) and (11). The second step is to solve first order equations and the third step is to solve the second order equations. The SIMPLE algorithm is used for each step. The final solution is the summation of $U_{0}, U_{1}$ and $U_{2}$. A three-part computer program has been provided for solving the three set of discretized equations. A uniform $300 \times 50$ staggered grid is employed for the present study. The boundary conditions at the channel inlet are:

$$
\begin{aligned}
& \left.u(y)\right|_{x=0}=\left.u_{0}(y)\right|_{x=0}=u_{i n} \\
& \left.u_{1}(y)\right|_{x=0}=\left.u_{2}(y)\right|_{x=0}=0 \\
& \left.T(y)\right|_{x=0}=\left.T_{0}(y)\right|_{x=0}=T_{i n} \\
& \left.T_{1}(y)\right|_{x=0}=\left.T_{2}(y)\right|_{x=0}=0
\end{aligned}
$$




\section{AnAlytic Solutions for Fully DEVELOPED FLOW in CHANNEL}

\subsection{Poiseuille Number using Second Order Model}

At high Knudsen number, no-slip boundary condition is not a good assumption and slip boundary conditions must be used. For isothermal flows with tangential momentum accommodation coefficient $\sigma_{v}=1$, for analytical solution we use the general second-order slip condition with the following dimensionless form (Karniadakis and Beskok (2004)):

$U_{s}-U_{w}=C_{1} K n\left(\frac{\partial U}{\partial n}\right)-C_{2} K n^{2}\left(\frac{\partial^{2} U}{\partial n^{2}}\right)$

The $C_{1}$ and $C_{2}$ are the slip constant coefficients. Different researcher developed this coefficients and in Table 1some of them shown. More information is available in (Karniadakis and Beskok (2004)). The analytical slip velocity profile is obtained using momentum equation and the above slip model as follows:

$$
\begin{aligned}
u(y)=\frac{-6 u_{m}}{\left[1+6 \frac{2-\sigma_{v}}{\sigma_{v}}\right.} & \left.\left(C_{1} K n+2 C_{2} K n^{2}\right)\right] \\
& \times\left[\left(\frac{y}{H}\right)^{2}-\left(\frac{y}{H}\right)\right. \\
& \left.-\frac{2-\sigma_{v}}{\sigma_{v}}\left(C_{1} K n+2 C_{2} K n^{2}\right)\right]
\end{aligned}
$$

Poiseuille number for fully developed flow is:

$P o=C f . R e=\frac{24}{\left(1+6 C_{1} K n+12 C_{2} K n^{2}\right)}$

$C_{f}$ is wall friction coefficient.

Table 1 Coefficients for slip models (Karniadakis and Beskok (2004))

\begin{tabular}{ccc}
\hline Author(s) & $\mathrm{C}_{1}$ & $\mathrm{C}_{2}$ \\
\hline Cercignani (Cercignani and Daneri 1963) & 1.1466 & 0.9756 \\
Revised Cercignani (Hadjiconstantinou 2003) & 1.1466 & 0.647 \\
Deissler (1964) & 1 & $9 / 8$ \\
Hisa and Domoto (1983) & 1 & 0.5 \\
Schamberg (1947) & 1 & $5 \pi / 12$ \\
Maxwell (Kennard 1938) & 1 & 0 \\
General form & 1 & -0.5 \\
\hline
\end{tabular}

\subsection{Analytical Solution using the Perturbation Methods for the Fully Developed Flow}

For the fully developed flow, $v=0, u=u(y)$ and $P=P(x)$. Therefore:

$v_{0}=v_{1}=v_{2}=0$

$u_{0}=u_{0}(y), u_{1}=u_{1}(y), u_{2}=u_{2}(y), \ldots$

$P_{0}=P_{0}(x), P_{1}=P_{1}(x), P_{2}=P_{2}(x), \ldots$

Hence, different orders of tangential momentum equations are solved with the corresponding boundary conditions. For incompressible flow, slip does not change the flow rate. That is:

$\dot{m}=\dot{m}_{0}, \dot{m}_{1}=\dot{m}_{2}=0$
For fully developed region we solve momentum equations using perturbation expansion for every order of $K n$, sothe zeroth order of velocity and its corrections become:

$$
\begin{aligned}
u_{0}(y)=-6 u_{m}\left[\left(\frac{y}{H}\right)^{2}-\left(\frac{y}{H}\right)\right] \\
u_{i}(y)=\left(-6 \frac{2-\sigma_{v}}{\sigma_{v}}\right)^{i-1} \times\left(i-1+6 \frac{2-\sigma_{v}}{\sigma_{v}}\right) \\
\times u_{m}\left\{6\left[\left(\frac{y}{H}\right)^{2}-\left(\frac{y}{H}\right)\right]\right. \\
+1\} \quad i \geq 1
\end{aligned}
$$

$i$ is level or order number in perturbation expansion. The final slip velocity profile is obtained by substituting the Eq. (16) into the perturbation expansion of the velocity:

$$
\begin{aligned}
& u(y)=\sum_{i=0}^{n} u_{i}(y)(K n)^{i} \\
& =\left(\frac{-6 u_{m}}{\left[1+6 \frac{2-\sigma_{v}}{\sigma_{v}}\left(K n-K n^{2}\right)\right]}\right) \\
& \times\left[\left(\frac{y}{H}\right)^{2}-\left(\frac{y}{H}\right)-\frac{2-\sigma_{v}}{\sigma_{v}}\left(K n-K n^{2}\right)\right]
\end{aligned}
$$

We use $C_{1}$ and $C_{2}$ for investigate effects of this coefficients on the analytical solution of Perturbation Method and compare with results of other researcher. If we use Eq.(17) to obtain Poiseuille number, the result is Eq.(13) with $C_{l}=1$ and $C_{2}=-0.5$.

\section{NUMERICAL RESULTS AND DISCUSSION}

To assure that each numerical result is sufficiently accurate, grid resolution studies have been performed for each $\mathrm{Po}$ and $\mathrm{Nu}$. Fully developed $\mathrm{Nu}$ and $\mathrm{Po}$ number for $R e=1, P r=0.7$ and $K n=0.06$, obtained from different grids, are shown in Table 2. For these results we used slip-coefficients that proposed by Hisa and Domoto (Karniadakis and Beskok (2004)). As shown in Table 2, a grid with $300 \times 50$ resolution, is suitable, thus the following results are based on this grid size.

Table 2. Grid study results

\begin{tabular}{ccc}
\hline Grid resolution & $N u$ & $P o$ \\
\hline $\mathbf{2 0 0} \times \mathbf{2 0}$ & 6.193 & 17.629 \\
$\mathbf{3 0 0} \times \mathbf{3 0}$ & 6.218 & 17.694 \\
$\mathbf{3 0 0} \times \mathbf{5 0}$ & 6.236 & 17.735 \\
$\mathbf{4 0 0} \times \mathbf{6 0}$ & 6.237 & 17.737 \\
\hline
\end{tabular}

\subsection{Hydrodynamics}

\subsubsection{Comparison Analytical Solution with Numerical Solution using Perturbation Method}

As mentioned and shown in Fig.1, the velocity and temperature profiles are uniform at inlet of the channel. In Fig. 2, Dimensionless form of the no-slip velocity profile and the velocity corrections are compared with the analytical solution at once of the sections that the flow is fully developed.As shown, good agreement isfound between the analyticaland numerical results. In Fig.3, the slip velocity, no-slip velocity and 

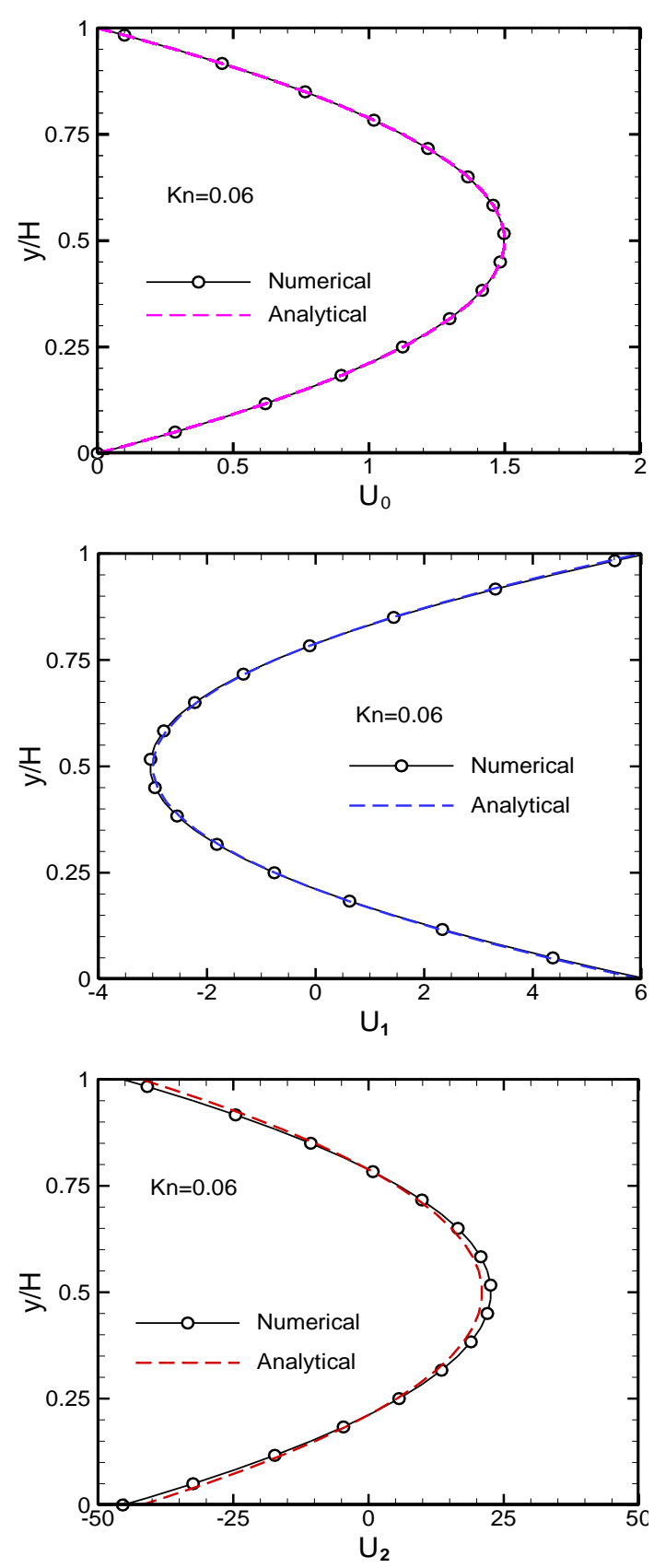

Fig. 2. Zeroth, first and second order of corrections velocity

correctional velocities are compared. Zeroth order of velocity or noslip boundary condition velocity profile $\left(U_{0}\right)$ is corrected by first order $\left(U_{l}\right)$ and second order $\left(U_{2}\right)$ corrections to obtain slip velocity profile $(U)$.

\subsubsection{Comparison Analytical and Numerical Solution with other Slip Models}

In Fig. 4, the slip and no-slip fully developed velocity profiles obtained by the numerical solution (using perturbation method) and slip velocities obtained by the Eq. (12) are shown. Good agreement isfound between the results. For a better comparison between the perturbation method results and the results produced by different slip models, Fig. 5 shows normalized slip

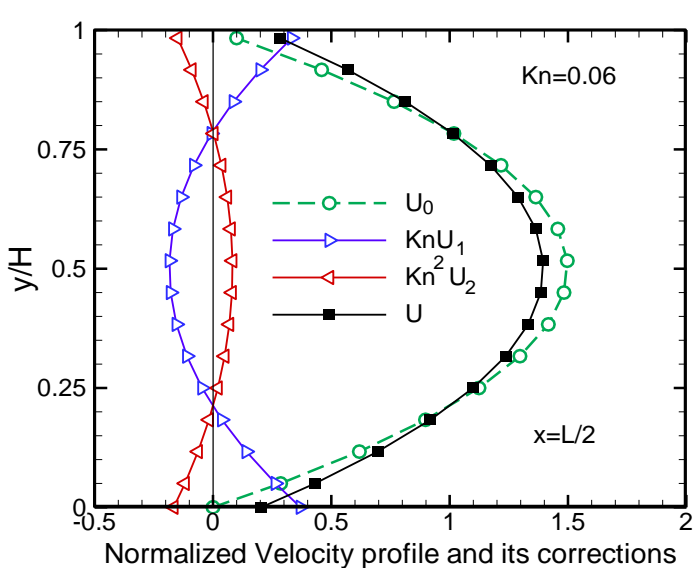

Fig.3. Fully developed dimensionless slip velocity profile, no-slip velocity and correctional velocities for $K n=0.06$.

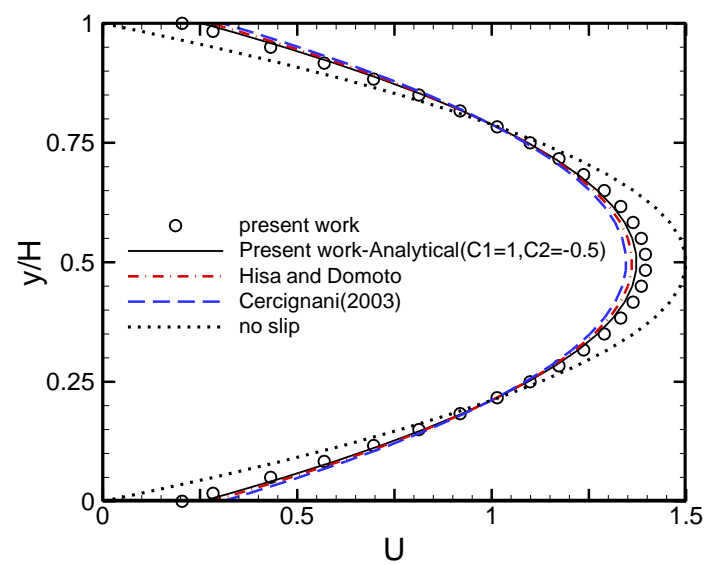

Fig. 4. Slip and no-slip velocities for $K n=0.06$.

velocities at the channel walls versus the Knudsen number. If we use only the first order perturbation expansions $\left(\varphi=\varphi_{0}+K n \varphi_{1}\right)$, the slip velocity is overpredicted as Knudsen number increased. Also, if we use first and second order perturbation expansions $\left(\varphi=\varphi_{0}+K n \varphi_{1}+K n^{2} \varphi_{2}\right)$, with $C_{1}=1$ and $C_{2}=-0.5$ the slip velocity is underpredicted as Knudsen number is increased. However, if we use other slip coefficients such as those proposed by Hisa and Domoto, the model is more reliable compared to other available data. Numerical results of perturbation method deviate from the other results as $K n$ is increased. This reveals that more corrections are needed in the perturbation method as $K n$ is increased.

In Fig. 6 the result of numerical solution for $P o$ and analytical solution using Eq. (13) for all slip coefficients that presented in(Karniadakis and Beskok (2004)) are shown. In Fig.6 it is shown that fully developed Po with Diessler's slip coefficients has a good agreement with corresponding analytical solutions. Also it is seen that $P o$ decreases with increasing in $K n$. 


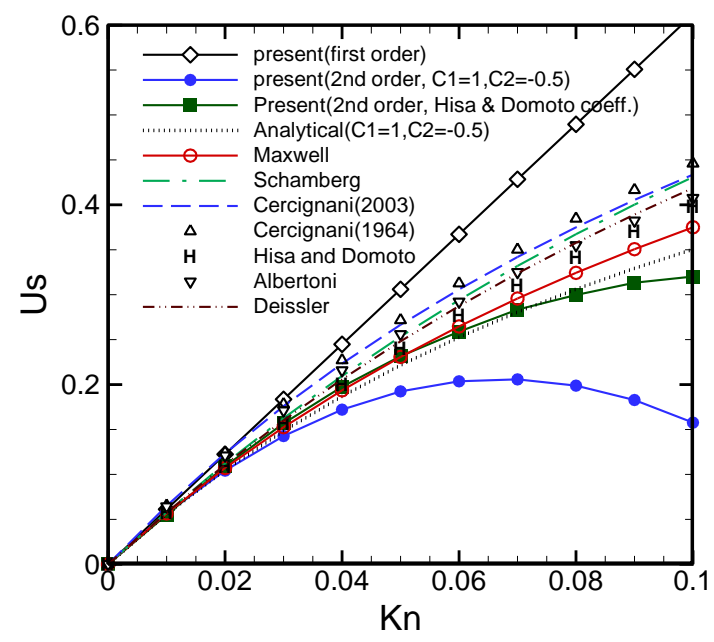

Fig. 5. Dimensionless slip velocity versus $K n$ for the Poiseuille flow.

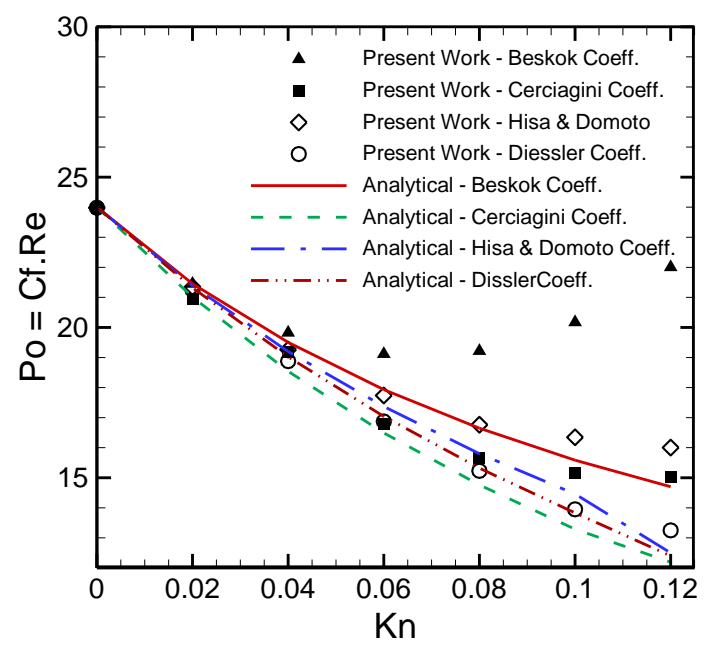

Fig. 6. Variation of fully developed Powith Knudsen number, for $\boldsymbol{R e}=\mathbf{1}$.

\subsection{Heat Transfer}

\subsubsection{Constant Wall Heat Flux Boundary Condition (CWHF)}

The zeroth to second order perturbation equations for Constant Wall Heat Flux (CWHF) with $C_{I}=1$ and $C_{2}=-0.5$ are solved numerically using SIMPLE algorithm. Finally the total temperature $\left(T=T_{0}+\right.$ $\left.K n T_{1}+K n^{2} T_{2}\right)$ is calculated. Dimensionless temperature profiles that are defined in Eq. (18) for jump and no-jump conditions are shown in Fig.7.This figure is for a section that the flow is thermally fully developed

$\theta=\frac{\left(T_{w}-T\right)}{\left(T_{w}-T_{m}\right)}$

In Fig.8, Nusselt numbers obtained with different models and for different $K n$ are shown and compared with results of Aydinand Avci (2007) and Inman(1964). Then different proposed values for $C_{2}$ when $C_{1}=1$ are

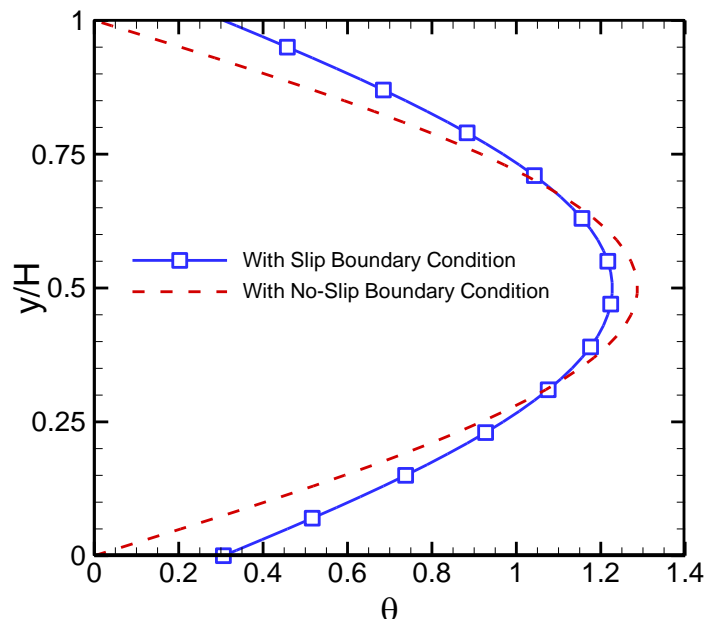

Fig.7. Fully developed dimensionless temperature profiles for slip and no-slip conditions for $\operatorname{Re}=1$, $P r=0.7$ and $K n=0.06$

tested. The results are in Fig. 8 and Table 3. It is found that for low $K n$, there is a good agreement between $\mathrm{Nu}$ that obtained in present study and $\mathrm{Nu}$ values obtained by Aydinand Avci (2007) and Inman (1964). When Kn increases, the difference between $\mathrm{Nu}$ obtained in this study and other results is increased.Fig. 8 demonstrates that $N u$ decreases with increased of $K n$.

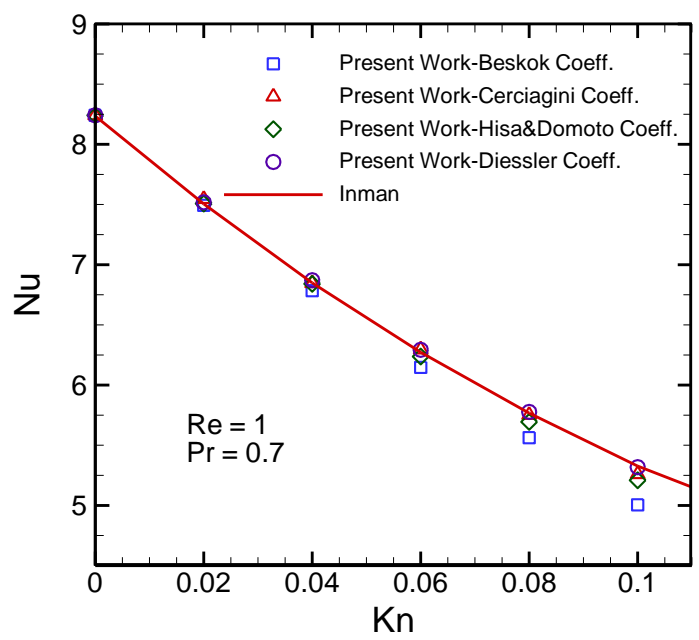

Fig. 8. Variation of fully developed $\mathrm{Nu}$ for $\mathrm{CWHF}$ case with $K n$ when $\operatorname{Re}=1, P r=0.7$

Figure 9 shows that the local Nusselt number decreases when proceeding along the channel. In this figure, it is shown that Nusselt number decreases rapidly at inlet of the channel and then tends to a constant value and this is show developing of flow. $\left.(\partial T / \partial y)\right|_{w}$ And $\left(T_{w}-T_{m}\right)$ both decrease rapidly near the inlet of channel but $\left.(\partial T / \partial y)\right|_{w}$ decrease much more rapidly than $\left(T_{w}-T_{m}\right)$, so Nusselt number decreases at first. One can understand from Fig. 9 that if $K n$ increases then thermal entry length increases. It is noticeable that this Figure is for $R e=1$ and we can expect larger thermal entry length in larger Reynolds numbers. 
Table 3. Fully developed Nu values for $P r=0.7, R e=1$ for the CWHF

\begin{tabular}{ccccccc}
\hline$K n$ & $\begin{array}{c}\text { Present work } N u \\
\text { number } \\
\text { using Beskok } \\
\text { Coeff. }\end{array}$ & $\begin{array}{c}\text { Present work } N u \\
\text { number using } \\
\text { Cercignani } \\
\text { Coeff. (1964) }\end{array}$ & $\begin{array}{c}\text { Present work } N u \\
\text { number using } \\
\text { Hisa\&Domoto } \\
\text { coeff. (1983) }\end{array}$ & $\begin{array}{c}\text { Present work } N u \\
\text { number using } \\
\text { Diessler Coeff. } \\
(1964)\end{array}$ & $\begin{array}{c}N u \text { number } \\
\text { Inman. (1964) }\end{array}$ & $\begin{array}{c}N u \text { number } \\
\text { Aydin. (2007) }\end{array}$ \\
\hline 0.0 & 8.241 & 8.241 & 8.241 & 8.241 & 8.235 & 8.236 \\
0.02 & 7.493 & 7.546 & 7.508 & 7.518 & 7.505 & 7.5 \\
0.04 & 6.784 & 6.847 & 6.841 & 6.871 & 6.849 & 6.842 \\
0.06 & 6.147 & 6.296 & 6.237 & 6.293 & 6.272 & 6.262 \\
0.08 & 5.563 & 5.752 & 5.694 & 5.777 & 5.767 & 5.756 \\
0.1 & 5.005 & 5.260 & 5.209 & 5.318 & 5.327 & 5.314 \\
\hline
\end{tabular}

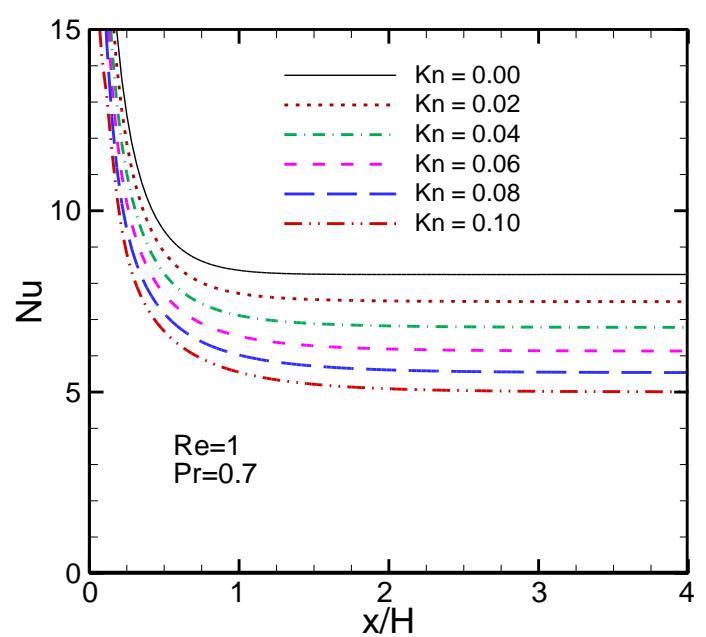

Fig. 9. Axial variation of local $\mathrm{Nu}$ for $\mathrm{CWHF}$ case at $\operatorname{Re}=1, \operatorname{Pr}=0.7$

\subsubsection{Constant Wall Temperature Solution(CWT)}

Axial variation of temperature is not linear in constant wall temperature (CWT) boundary condition. Therefore axial conduction can be important for CWT cases even if the flow is thermally fully developed. The significance of streamwise conduction is generally established by the magnitude of the flow Peclet number. The Peclet number, $P e=R e . P r$, represents the ratio of the thermal energy convected to the fluid, to the thermal energy axially conducted within the fluid. A low $P e$, which is common for micro flows, generally indicates that streamwise conduction effects must be considered. Since the Pein microchannels as much lower than 100 the effects of axial conduction should be taken into account. In fact we except that for low $P e$, contribution of axial conduction is considerable compared to convection heat transfer. $N u$ is lower than when axial conduction is negligible. The variation of $\mathrm{Nu}$ with $\mathrm{Kn}$ for different values of $P e$ for Constant Wall Temperature (CWT) is shown in Fig.10, in this figure it shown that for a fixed $P e, N u$ decreases as $K n$ is increased. Fig. 10 shows effects of $\mathrm{Pe}$ on $\mathrm{Nu}$ in different $K n$. This figure shows good agreement between our numerical results and results presented by Hadjiconstantinou (2003). This figure shows that the $\mathrm{Nu}$ decreases monotonically with increasing $\mathrm{Kn}$ at constant $\mathrm{Pe}$. The effect of axial heat conduction is to increase the $N u$ throughout the slip flow regime. When rarefaction is increasing in $K n$, velocity slip increased so convection heat transfer and $N u$ are decreased.

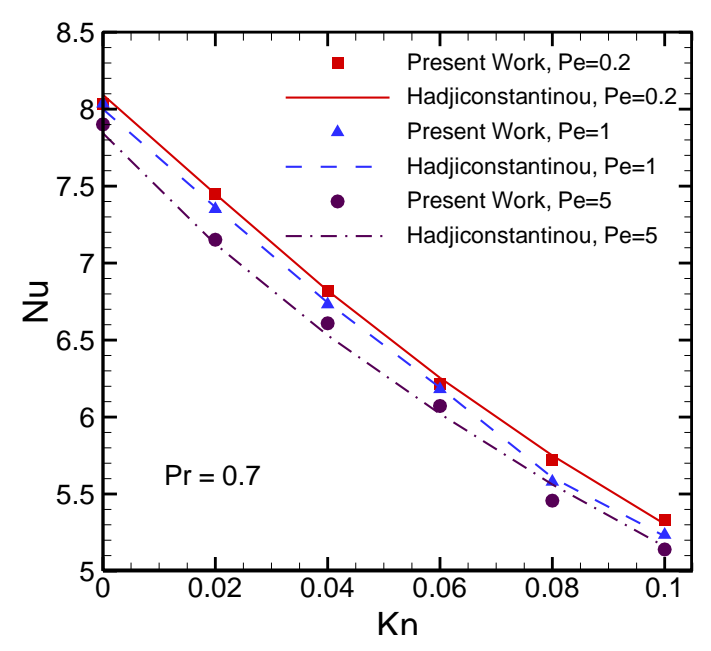

Fig. 10. Variation of fully developed $N u$ number with $K n$ number for $P e=0.2, P e=1$ and $P e=5$.

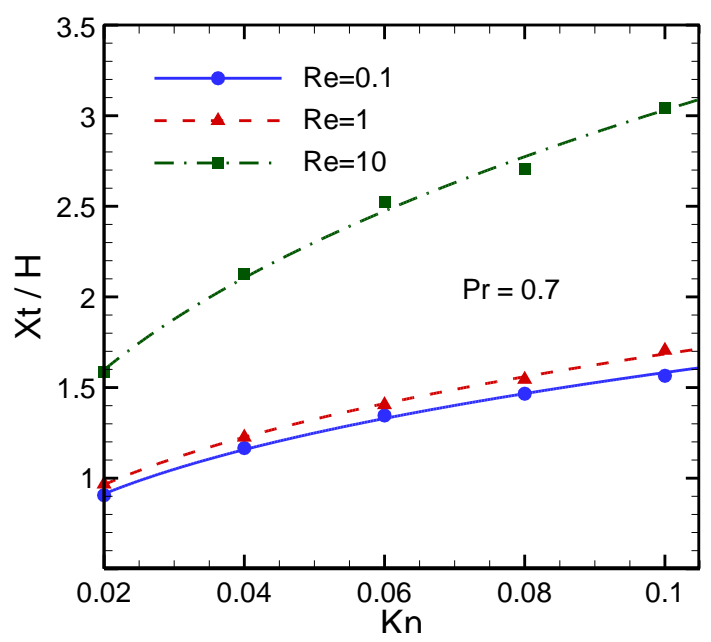

Fig. 11. Variation of thermal entrance length with Knudsen number for $R e=10, R e=1, R e=0.1$ and $\operatorname{Pr}=0.7$

Figure 11 shows thermal entrance length for CWT case, with increasing of rarefaction, thermal entrance length is increased. Also when $R e$ is increased, the thermal entrance length is also increased.

Figure 12 shows dimensionless slip velocity $\left(U_{s}\right)$ and temperature jump $\left[\left(T_{w}-T_{s}\right) /\left(T_{m}-T_{s}\right)\right]$ in fully developed flow region. This figure show that velocity slip and temperature jump increasing when rarefaction is increased. Also in Fig.13dimensionless slip velocity and temperature jump for CWT case on wall across the channel are shown for $R e=1$ and $P r=0.7$. 


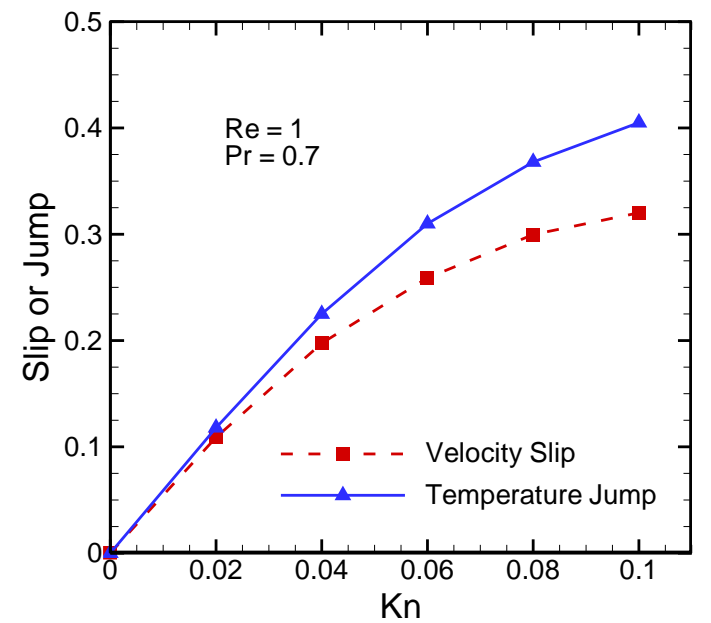

Fig. 12. Velocity slip and temperature jump on wall for $\operatorname{Re}=1$ and $\operatorname{Pr}=0.7$

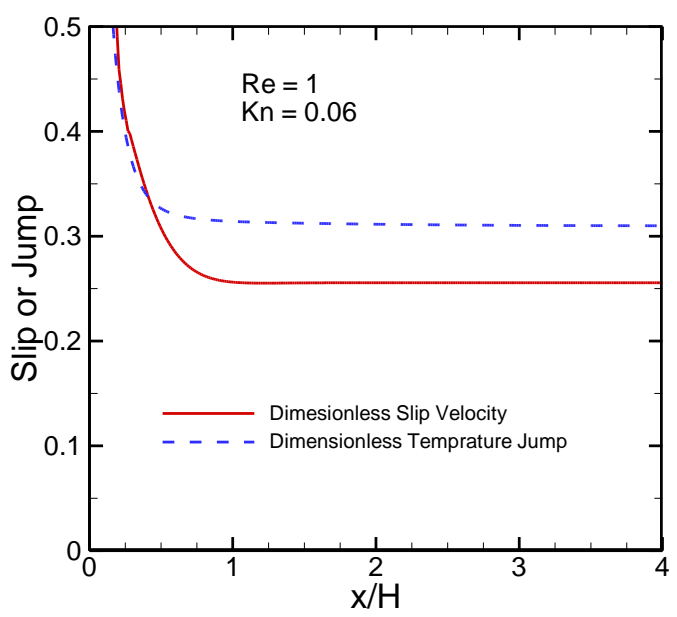

Fig. 13. Velocity slip and temperature jump on wall along the channel length

\subsection{The Role of the Accommodation Coefficients on Heat Transfer Rate}

Momentum and energy transfer between the gas molecules and the surface require specification of interactions between the impinging gas molecules and the surface. A detailed analysis of this is quiet complicated and requires complete knowledge of the scattering kernels. The thermal and momentum accommodation coefficient $\mathrm{s}, \sigma_{T}$ and $\sigma_{v}$, are defined by

$\sigma_{T}=\frac{d E_{i}-d E_{r}}{d E_{i}-d E_{w}} \quad, \quad \sigma_{v}=\frac{\tau_{i}-\tau_{r}}{\tau_{i}-\tau_{w}}$

$d E_{i}, d E_{r}$ denote energy fluxes of incoming and reflected molecules per unit time respectively and $d E_{w}$ denotes the energy fluxes if the all incoming molecules had been reemitted with the energy flux corresponding to the surface temperature, $T_{w}$ (Karniadakis and Beskok (2004)).
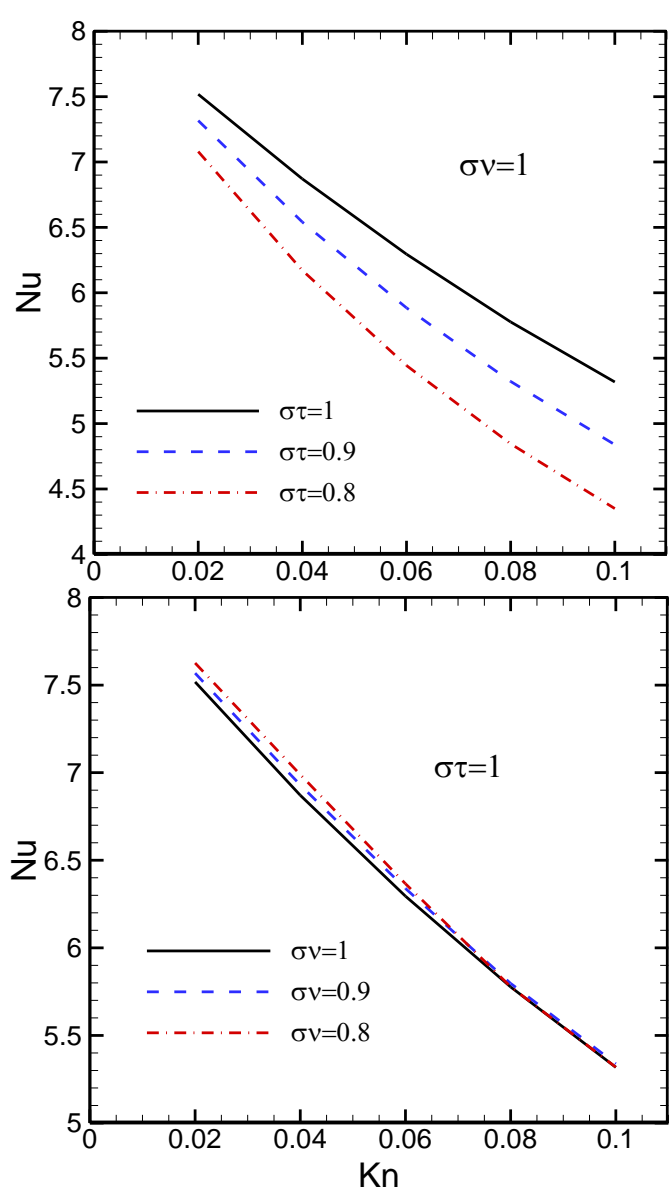

Fig. 14. Variation of $N u$ against $K n$ for various values of thermal and momentum accommodation coefficients

The effect of accommodation coefficients on heat transfer is shown in Fig.14. Nu is increasing with increasing in thermal accommodation coefficient and decreasing in momentum accommodation coefficient.

\subsection{The Effect of Thermal Creep on Flow and Heat Transfer}

Thermal creep occurs in gas flows when axial temperature gradients are applied along the channel walls Sone (2002). In channel flows, this boundary treatment induces a tangential creep velocity $\left(u_{c}\right)$ in the interior gas close to the walls, such that the gas flows in the direction of the temperature gradient. The following thermal creep $u_{c}$ should be added to slip velocity

$u_{c}=\frac{3}{4} \frac{\mu}{\rho T_{w}}\left(\frac{\partial T}{\partial x}\right)_{\text {wall }}$

This case is investigated in present work when constant heat flux (CWHF) boundary condition is applied. The effects of thermal creep flow on fully developed $P o$ and $\mathrm{Nu}$ are shown in Fig.15 and Fig.16 for $P e=0.5$ and $u_{c} / u_{m}=+0.25$ (that means thermal creep is in streamwise direction). The results are compared with the available results of Van Rij et al. (2007). As shown in Fig.15 and 16, if thermal creep flow has a same direction with streamwise $\left(u_{c}>0\right.$ or $\left.(\partial T / \partial x)_{w}>0\right)$ then it increases Nusselt number and decreases Poiseuille number due to increasing slip in walls. 


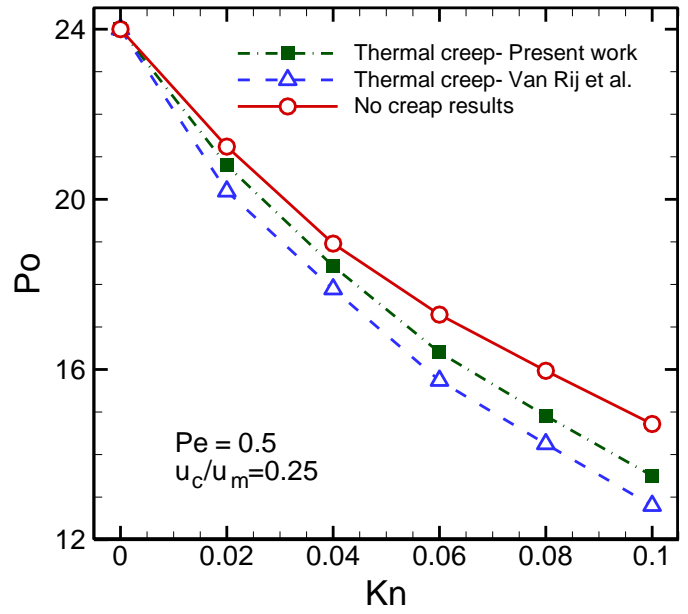

Fig. 15. Effect of thermal creep flow on Poiseuille number for various values of Knudsen number

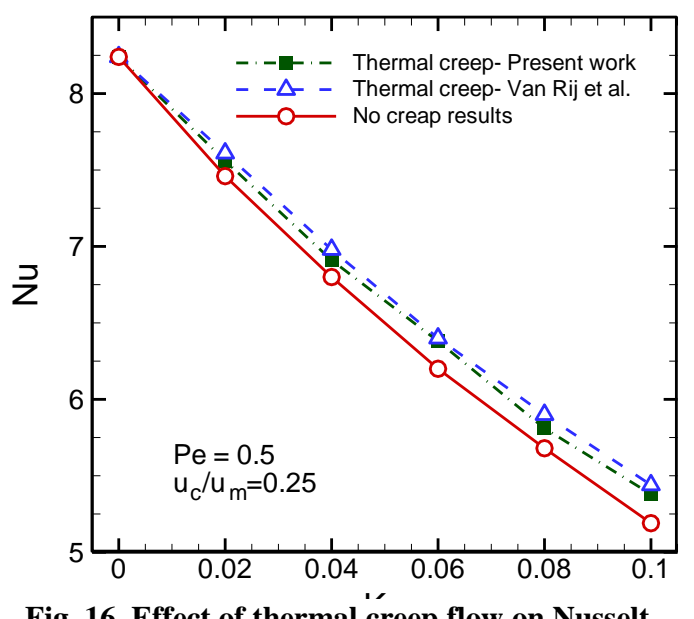

Fig. 16. Effect of thermal creep flow on Nusselt number for various values of Knudsen number

\section{Conclusions}

In this study slip-flow heat transfer in a laminar, steady state, two-dimensional incompressible flow between parallel plates micro-channel is investigated numerically using perturbation method. Both hydrodynamically and thermally developing flow cases are examined. The Poiseuille and Nusselt numbers for 2-D microchannels with both constant wall heat flux (CWHF) and constant wall temperature (CWT) thermal boundary conditions for the slip flow regime have been numerically investigated. The resulting $P o$, and $\mathrm{Nu}$ include the effects of second-order velocity slip and temperature jump boundary conditions. In this study the effects of viscous dissipation and compressibility are neglected. The interactive effect of the rarefaction on Nusselt number has been studied and found that the Nusselt number decreases when rarefaction increases. Also thermal entrance length is investigated and it is found that increasing in $R e$ or $K n$, leads to increase in thermal entrance length. For CWT case, $P e$ has a significant role in heat transfer rate, for low $P e$, thus we should take in to account the effects of axial conduction. Effects of axial conduction increase with decreasing $P e$ and decrease with increasing of rarefaction.

At the end, it is illustrated that when the effect of thermal creep is accounted, if thermal creep has the same direction with streemwise, since the slip velocity at the wall increases, the momentum transfer increases and so Nusselt number increase.

Finally one can conclude that perturbation method is capable to simulate slip flow heat transfer in micropoiseuille flow

\section{REFERENCES}

Ameel, T.A., X.M. Wang, R.F. Barron, R.O. Warrington, (1997).Laminar forced convection in a circular tube with constant heat flux and slip flow, Microscale Thermal Engineering, 1 (4) 303-320.

Aydin, O., M. Avci, (2007).Analysis of laminar heat transfer in micro-poiseuille flow, International Journal of Thermal Science, 46 30-37.

Barron, R.F., X.. Wang, R.O. Warrington, T. Ameel, (1996).Evaluation of the eigenvalues for the Graetz problem in slip-flow, Int. Comm. Heat Mass Transfer, 23 (4) 563-574.

Barron, R.F., X. Wang, T.A. Ameel, R.O. Warrington, (1997).The Graetz problem extended to slip-flow, International Journal of Heat and Mass Transfer, 40 (8) 1817-1823.

Beskok, A., G.E. Karniadakis, (1994).Simulation of heat and momentum transfer in complex microgeometries, Journal of Thermophysics Heat Transfer, 8 355-370.

Hadjiconstantinou, N. G., (2003).Comment on Cercignani's second-order slip coefficient, Phys. Fluids, Vol. 15, pp. 2352-2354,

Hadjiconstantinou, N.G., O., Simek,(2002).Constantwall-temperature Nusselt Number in Micro and Nano Channels. Journal of Heat Transfer, 124, 356-364,

Inman, R.M.,(1964) Laminar slip flow heat transfer in a parallel-plate channel or a round tube with uniform wall heating, NASA TD-2393.

Karniadakis, G., A. Beskok, N. Aluru, ,(2004).Microflows and Nanoflows Fundamentals and Simulations, Springer

Morini, G.L.,(2004).Single-phase convective heat transfer in microchannels: a review of experimental results, International Journal of Thermal Sciences 43631-651

Obot, N.T.,(2002).Toward a better understanding of friction and heat/mass transfer in microchannelsA literature review, Microscale Thermophysical Engineering, 6 155-173. 
M. Saghafian et al. /JAFM, Vol. 8, No. 1, pp. 123-132, 2015.

Palm, B.,(2001).Heat transfer in microchannels, Microscale Thermophysical Engineering, 5 155175.

Qin, F. H., D. J., Sun, and X. Y., Yin, (2007) Perturbation analysis on gas flow in a straight microchannel, Physics of fluids, Vol. 19, pp 027103-14,

Sobhan, C.B.,S.V. Garimella, (2001).A comparative analysis of studies on heat transfer and fluid flow in microchannels, Microscale Thermophysical Engineering, 5 293-311.

Sone, Y., (2002).Kinetic Theory and fluid dynamics, Birkhauser, Boston.
Sparrow, E.M., S.H. Lin, (1962).Laminar heat transfer in tubes under slip-flow conditions, J. Heat transfer, 84 (4) 363-369

Tunc, G. Y., Bayazitoglu,(2001).Heat transfer in microtubes with viscous dissipation, International Journal of Heat and Mass Transfer, 44 23952403.

Van Rij, J.V., T., Harman, T. and Ameel, (2007).The effect of creep flow on two-dimensional isoflux microchannels, International Journal of Thermal Sciences, 46, 1095-1103. 